\title{
PACS-CS results for 2+1 flavor lattice QCD simulation on and off the physical point
}

\section{Yoshinobu Kuramashi* for the PACS-CS Collabolation}

Center for Computational Sciences and Graduate School of Pure and Applied Sciences,

University of Tsukuba, Tsukuba, Ibaraki 305-8571, Japan

We report on the PACS-CS project focusing on a direct simulation of $2+1$ flavor QCD on the physical point and chiral analysis of meson and baryon masses off the physical point with both the SU(2) and SU(3) chiral perturbation theories. Configurations are generated with the $O(a)$ improved Wilson quark action and the Iwasaki gauge action. The up-down quark is simulated by employing the DDHMC algorithm with several improvements and the UV-filtered PHMC algorithm is implemented for the strange quark. We investigate the convergence behaviors of the SU(2) and SU(3) chiral expansions up to NLO for the pseudoscalar meson sector, where the updown quark mass ranges from $3 \mathrm{MeV}$ to $24 \mathrm{MeV}$ and the strange quark mass is chosen around the physical value. The fit results for the low energy constants are compared with those recently obtained by other groups. We also discuss the importance of the direct simulation at the physical point by comparing the physical quantities measured on the physical point with those estimated by the extrapolation method.

The XXVI International Symposium on Lattice Field Theory

July 14-19 2008

Williamsburg, Virginia, USA

\footnotetext{
*E-mail: kuramasi@het.ph.tsukuba.ac.jp
} 


\section{Introduction}

The aim of lattice QCD calculation is to nonperturbatively evaluate physical quantities from first principles with the systematic errors under control. Most troublesome are quenching effects and uncertainties associated with chiral extrapolation, which should be removed by $2+1$ flavor simulation at the physical point.

The previous CP-PACS/JLQCD project[1] focuses on performing $N_{f}=2+1$ lattice QCD simulations incorporating the dynamical strange quark by the Polynomial Hybrid Monte Carlo (PHMC) algorithm[2], where the nonperturbatively $O(a)$-improved Wilson quark action[3] and the Iwasaki gauge action[4] are employed on a $(2 \mathrm{fm})^{3}$ lattice with the up-down quark mass down to $67 \mathrm{MeV}$. This project is succeeded by the PACS-CS (Parallel Array Computer System for Computational Sciences) project $[5,6,7,8,9,10,11,12,13,14]$, which aims at the physical point simulation with the same quark and gauge actions as the CP-PACS/JLQCD project on enlarged physical volumes. We reduce the up-down quark masses using the domain-decomposed HMC (DDHMC) algorithm armored with several improvements and implement the UV-filtered PHMC algorithm[8] for the strange quark.

In this report we demonstrate the feasibility of a direct simulation at the physical point and examine viability of extrapolation method with the chiral perturbation theories. We apply the $\mathrm{SU}(2)$ and $\mathrm{SU}(3)$ chiral perturbation theories (ChPTs) to the pseudoscalar meson masses and the decay constants with the up-down quark mass ranging from $3 \mathrm{MeV}$ to $24 \mathrm{MeV}$ and two choices of the strange quark mass around the physical value. The low energy constants in the SU(2) and SU(3) ChPTs are determined and compared with those obtained with the different quark and gauge actions employed by other groups. We discuss the convergence behaviors of the SU(2) and SU(3) chiral expansions up to NLO. We also investigate the quark mass dependence of the nucleon mass employing the SU(2) heavy baryon chiral perturbation theory up to NNLO. We finally make a comparison between the physical quantities directly measured on the physical point and those estimated by the extrapolation method. This comparison reveals the necessity and the effectiveness of the direct simulation at the physical point.

All the calculations are done using the PACS-CS computer with a total peak speed of 14.3 TFLOPS developed and installed at University of Tsukuaba on 1 July 2006. A part of the results are already reported in Ref. [12, 13, 14].

\section{Simulation details}

Our simulation is carried out using the $O(a)$-improved Wilson quark action with a nonperturbative improvement coefficient $c_{\mathrm{SW}}=1.715$ [3] on a $32^{3} \times 64$ lattice. The lattice spacing is found to be $0.0907(13) \mathrm{fm}$ whose determination is explained later. Simulation parameters are summarized in Table 1. We choose seven combinations of the hopping parameters $\left(\kappa_{\mathrm{ud}}, \kappa_{\mathrm{s}}\right)$ based on the analysis of the previous CP-PACS/JLQCD results. We employ two choices of $\kappa_{\mathrm{s}}$ to investigate the strange quark mass dependences. The heaviest combination $\left(\kappa_{\mathrm{ud}}, \kappa_{\mathrm{s}}\right)=(0.13700,0.13640)$ corresponds to the lightest one in the previous CP-PACS/JLQCD project. We expect that the combination $\left(\kappa_{\mathrm{ud}}, \kappa_{\mathrm{s}}\right)=(0.137785,0.13660)$ would be the physical point, which is estimated at the early stage of our analyses. 
Table 1: Simulation parameters. MD time is the number of trajectories multiplied by the trajectory length $\tau$.

\begin{tabular}{llccccc}
\hline$\kappa_{\mathrm{ud}}$ & $\kappa_{\mathrm{s}}$ & DDHMC & $\tau$ & $\left(N_{0}, N_{1}, N_{2}, N_{3}, N_{4}\right)$ & MD time & $m_{\pi} L$ \\
\hline \hline 0.13700 & 0.13640 & plain & 0.50 & $(4,4,10)$ & 2000 & 10.3 \\
0.13727 & 0.13640 & plain & 0.50 & $(4,4,14)$ & 2000 & 8.4 \\
0.13754 & 0.13640 & plain & 0.50 & $(4,4,20)$ & 2250 & 6.0 \\
& 0.13660 & plain & 0.50 & $(4,4,28)$ & 2000 & 5.7 \\
0.13770 & 0.13640 & plain & 0.25 & $(4,4,16)$ & 2000 & 4.3 \\
0.13781 & 0.13640 & MP & 0.25 & $(4,4,4 / 6,6)$ & 990 & 2.3 \\
0.137785 & 0.13660 & MP2 & 0.25 & $(4,4,2,4,4)$ & 1000 & 2.4 \\
\hline
\end{tabular}

The DDHMC algorithm[15] allows us to access the small up-down quark mass region closer to the physical point. It is employed for the simulation points with $\kappa_{\mathrm{ud}} \leq 0.13770$. We divide the full lattice into $8^{4}$ blocks which are used for a geometric separation of the up-down quark determinant into the UV and the IR parts. This separation makes possible to incorporate the multiple time scale integration scheme[16], which reduce the stimulation cost significantly. We employ the replay trick $[15,17]$ choosing the threshold $\Delta H>2$. The detailed description about the DDHMC algorithm is given in Refs. [12, 13].

Reducing the up-down quark mass, however, the IR force $F_{\mathrm{IR}}$ becomes less stable yielding spike-like fluctuations, which results in larger $\Delta H$ with higher replay rates. At the simulation point of $\left(\kappa_{\mathrm{ud}}, \kappa_{\mathrm{s}}\right)=(0.13781,013640)$ we incorporate the mass preconditioning $[18,19]$ to tame the fluctuations of the IR force, which is divided into the preconditioner $F_{\text {IR }}^{\prime}$ with a new hopping parameter $\kappa_{\text {ud }}^{\prime}$ and the preconditioned part $\tilde{F}_{\mathrm{IR}} \cdot \kappa_{\text {ud }}^{\prime}$ is parametrized as $\kappa_{\text {ud }}^{\prime}=\rho_{1} \kappa_{\text {ud }}$ with $\rho_{1}$ less than unity. The step sizes for $F_{\mathrm{G}}, F_{\mathrm{UV}}, F_{\mathrm{IR}}^{\prime}, \tilde{F}_{\mathrm{IR}}$ are controlled by four integers $\left(N_{0}, N_{1}, N_{2}, N_{3}\right)$ as $\delta \tau_{\mathrm{G}}=\tau /\left(N_{0} N_{1} N_{2} N_{3}\right), \delta \tau_{\mathrm{UV}}=\tau /\left(N_{1} N_{2} N_{3}\right), \delta \tau_{\mathrm{IR}}^{\prime}=\tau /\left(N_{2} N_{3}\right), \delta \tilde{\tau}_{\mathrm{IR}}^{\prime}=\tau / N_{3}$ with $\tau$ the trajectory length. This algorithm is refereed to as MPDDHMC. We choose $\delta \tau_{\mathrm{s}}=\delta \tau_{\mathrm{IR}}^{\prime}$ for the strange quark force in the UVPHMC algorithm based on our observation that $\left\|F_{\mathrm{S}}\right\| \approx\left\|F_{\mathrm{IR}}^{\prime}\right\|$.

At $\left(\kappa_{\mathrm{ud}}, \kappa_{\mathrm{s}}\right)=(0.13781,013640)$ we also implement several improvements for the inversion of the Wilson-Dirac operator on the full lattice. First one is the chronological guess for the initial solutions[20] with the use of the last 16 solutions. Second one is a nested BiCGStab solver consisting of the outer solver with double precision arithmetic and the inner one operated with single precision. The latter with an automatic stopping condition from $10^{-3}$ to $10^{-6}$ works as a preconditioner for the former. We employ a stringent tolerance $|D x-b| /|b|<10^{-14}$ for the outer solver to retain the reversibility of the molecular dynamics trajectories to high precision. Third one is the GCRO-DR (Generalized Conjugate Residual with implicit inner Orthogonalization and Deflated Restarting) algorithm[21] which is robust against the small eigen values of the Wilson-Dirac operator. It take over the inversion once the nested BiCGStab solver becomes stagnant.

For the run at $\left(\kappa_{\mathrm{ud}}, \kappa_{\mathrm{s}}\right)=(0.137785,013660)$ we apply twofold mass preconditioning (MP2DDHMC) to the IR force $F_{\mathrm{IR}}$, which is decomposed into $F_{\mathrm{IR}}^{\prime \prime}, \tilde{F}_{\mathrm{IR}}^{\prime}$ and $\tilde{F}_{\mathrm{IR}}$. Two hopping parameters are additionally introduced: $\kappa_{\mathrm{ud}}^{\prime \prime}=\rho_{2} \kappa_{\mathrm{ud}}^{\prime}=\rho_{2} \rho_{1} \kappa_{\mathrm{ud}}$ with $\rho_{1}$ and $\rho_{2}$ less than unity. We need five integers 
$\left(N_{0}, N_{1}, N_{2}, N_{3}, N_{4}\right)$ to adjust the step sizes for $F_{\mathrm{G}}, F_{\mathrm{UV}}, F_{\mathrm{IR}}^{\prime \prime}, \tilde{F}_{\mathrm{IR}}^{\prime}, \tilde{F}_{\mathrm{IR}}$. Our choice is $\delta \tau_{\mathrm{s}}=\delta \tau_{\mathrm{IR}}^{\prime \prime}$ for the UVPHMC algorithm.

\section{ChPT analyses on the pseudoscalar meson sector}

We examine the chiral behaviors of the pseudoscalar meson masses and the decay constants based on the SU(3) and SU(2) ChPTs up to NLO. Since the redefinition of some of the low energy constants (LECs) makes the one-loop expressions of the Wilson ChPT in terms of the AWI quark masses[22] equivalent to those in the continuum[12], we focus on the analyses with the use of the continuum ChPTs.

\subsection{SU(3) ChPT}

The one-loop expressions in the continuum SU(3) ChPT are given by[23]

$$
\begin{aligned}
\frac{m_{\pi}^{2}}{2 m_{\mathrm{ud}}} & =B_{0}\left\{1+\mu_{\pi}-\frac{1}{3} \mu_{\eta}+\frac{2 B_{0}}{f_{0}^{2}}\left(16 m_{\mathrm{ud}}\left(2 L_{8}-L_{5}\right)+16\left(2 m_{\mathrm{ud}}+m_{\mathrm{s}}\right)\left(2 L_{6}-L_{4}\right)\right)\right\} \\
\frac{m_{K}^{2}}{\left(m_{\mathrm{ud}}+m_{\mathrm{s}}\right)} & =B_{0}\left\{1+\frac{2}{3} \mu_{\eta}+\frac{2 B_{0}}{f_{0}^{2}}\left(8\left(m_{\mathrm{ud}}+m_{\mathrm{s}}\right)\left(2 L_{8}-L_{5}\right)+16\left(2 m_{\mathrm{ud}}+m_{\mathrm{s}}\right)\left(2 L_{6}-L_{4}\right)\right)\right\} \\
f_{\pi} & =f_{0}\left\{1-2 \mu_{\pi}-\mu_{K}+\frac{2 B_{0}}{f_{0}^{2}}\left(8 m_{\mathrm{ud}} L_{5}+8\left(2 m_{\mathrm{ud}}+m_{\mathrm{s}}\right) L_{4}\right)\right\} \\
f_{K} & =f_{0}\left\{1-\frac{3}{4} \mu_{\pi}-\frac{3}{2} \mu_{K}-\frac{3}{4} \mu_{\eta}+\frac{2 B_{0}}{f_{0}^{2}}\left(4\left(m_{\mathrm{ud}}+m_{\mathrm{s}}\right) L_{5}+8\left(2 m_{\mathrm{ud}}+m_{\mathrm{s}}\right) L_{4}\right)\right\}
\end{aligned}
$$

where we have six unknown LECs $B_{0}, f_{0}, L_{4,5,6,8} . \mu_{\mathrm{PS}}$ denotes the chiral logarithm defined by

$$
\mu_{\mathrm{PS}}=\frac{1}{16 \pi^{2}} \frac{\tilde{m}_{\mathrm{PS}}^{2}}{f_{0}^{2}} \ln \left(\frac{\tilde{m}_{\mathrm{PS}}^{2}}{\mu^{2}}\right)
$$

where

$$
\tilde{m}_{\pi}^{2}=2 m_{\mathrm{ud}} B_{0}, \quad \tilde{m}_{K}^{2}=\left(m_{\mathrm{ud}}+m_{\mathrm{s}}\right) B_{0}, \quad \tilde{m}_{\eta}^{2}=\frac{2}{3}\left(m_{\mathrm{ud}}+2 m_{\mathrm{s}}\right) B_{0}
$$

with $\mu$ the renormalization scale. The pseudoscalar meson decay constants are calculated with the nonperturbatively $O(a)$-improved axial vector current[24], though the renormalization factor is perturbatively evaluated up to one-loop level[25, 26]. We determine the LECs by a simultaneous fit of $m_{\pi}^{2} /\left(2 m_{\mathrm{ud}}\right), m_{K}^{2} /\left(m_{\mathrm{ud}}+m_{\mathrm{s}}\right), f_{\pi}$ and $f_{K}$ including the finite size corrections at one-loop level[27].

In Table 2 we compare our results for the LECs with the phenomenological estimates with experimental inputs[27, 28] and recent 2+1 flavor lattice QCD results[29, 30]. We observe that the situation is rather complex: Some LECs are consistent and others are not. To make the comparison easier we convert the SU(3) LECs to the SU(2) ones, where the number of LECs are reduced from six to four: $B, f, \bar{l}_{3}, \bar{l}_{4}$. We obtain $\bar{l}_{3}=3.47(11), \bar{l}_{4}=4.21(11)$ and $\bar{l}_{3}=3.50(11), \bar{l}_{4}=4.22(10)$ with and without the finite size corrections, respectively. These results are plotted in Fig. 1 together with the phenomenological estimates and the recent 2 and $2+1$ flavor lattice QCD results. We find that all the results reside in $3.0 \lesssim \bar{l}_{3} \lesssim 3.5$ and $4.0 \lesssim \bar{l}_{4} \lesssim 4.5$ except the MILC result for $\bar{l}_{3}$ which shows exceptionally small value. 
Table 2: Results for the LECs in the SU(3) ChPT together with the phenomenological estimates[27, 28] and the RBC/UKQCD[29] and the MILC results[30]. $f_{0}$ is perturbatively renormalized at one-loop level. $L_{4,5,6,8}$ are in units of $10^{-3}$ at the scale of $770 \mathrm{MeV}$.

\begin{tabular}{cccccc}
\hline \multicolumn{7}{c}{ PACS-CS } & phenomenology[27, 28] & RBC/UKQCD[29] & MILC[30] \\
& w/o FSE & w/ FSE & & & \\
\hline$f_{0}[\mathrm{GeV}]$ & $0.1160(88)$ & $0.1185(90)$ & 0.115 & $0.0935(73)$ & - \\
$f_{\pi} / f_{0}$ & $1.159(57)$ & $1.145(56)$ & 1.139 & $1.33(7)$ & $1.21(5)\left({ }_{-3}^{+13}\right)$ \\
$L_{4}$ & $-0.04(10)$ & $-0.06(10)$ & $0.00(80)$ & $0.139(80)$ & $0.1(3)\left({ }_{-1}^{+3}\right)$ \\
$L_{5}$ & $1.43(7)$ & $1.45(7)$ & $1.46(10)$ & $0.872(99)$ & $1.4(2)\left({ }_{-1}^{+2}\right)$ \\
$2 L_{6}-L_{4}$ & $0.10(2)$ & $0.10(2)$ & $0.0(1.0)$ & $-0.001(42)$ & $0.3(1)\left(\begin{array}{l}+2 \\
-3\end{array}\right)$ \\
$2 L_{8}-L_{5}$ & $-0.21(3)$ & $-0.21(3)$ & $0.54(43)$ & $0.243(45)$ & $0.3(1)(1)$ \\
\hline$\chi^{2} /$ dof & $4.2(2.7)$ & $4.4(2.8)$ & - & 0.7 & - \\
\hline
\end{tabular}
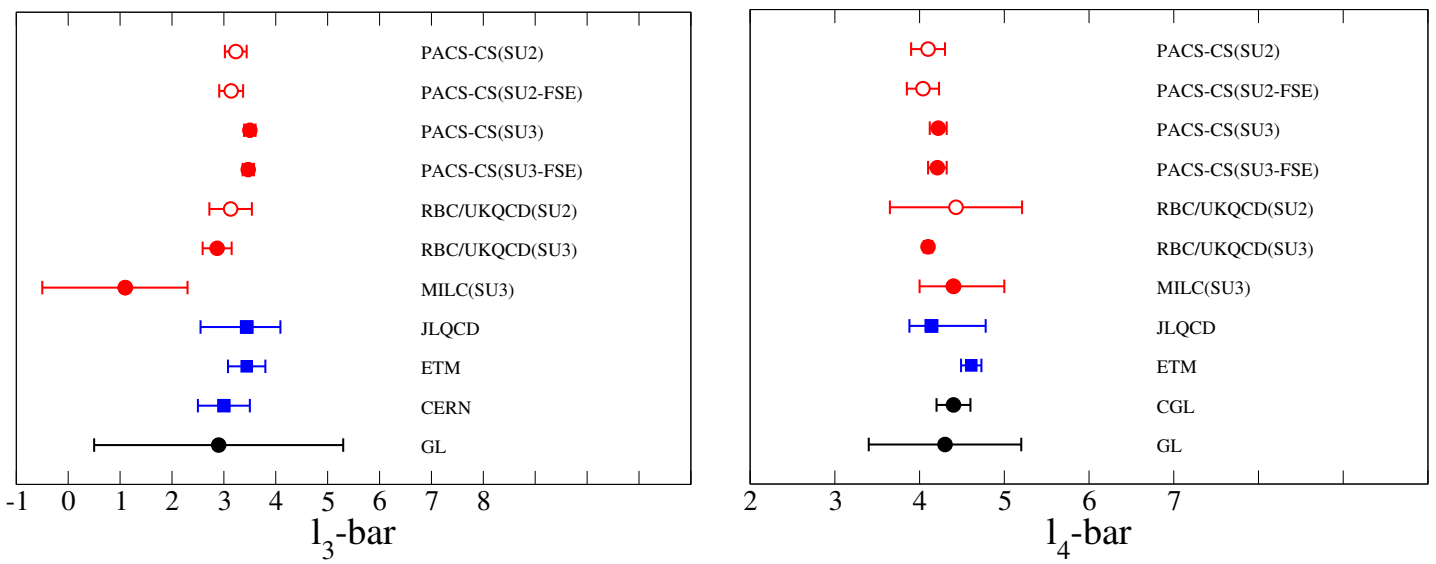

Figure 1: Comparison of the results for $\bar{l}_{3}$ and $\bar{l}_{4}$. Black symbols denote the phenomenological estimates[31, 32]. Blue ones are for 2 flavor results[33, 34, 35]. Red closed (open) symbols represent the results for the $\mathrm{SU}(3)(\mathrm{SU}(2))$ ChPT analyses in 2+1 flavor dynamical simulations[30, 29].

Although we obtain reasonable values for $\bar{l}_{3}$ and $\bar{l}_{4}$ in the SU(3) ChPT fit, there exists a concern that the values of $\chi^{2} /$ dof are unacceptably large. Its origin is found in the fit of $f_{\pi}$ and $f_{K}$ depicted in Fig. 2, where the strange quark mass dependence between the data at $\left(\kappa_{\mathrm{ud}}, \kappa_{\mathrm{s}}\right)=$ $(0.13754,0.13640)$ and $(0.13754,0.13660)$ is not properly described by the SU(3) ChPT. The convergence of the SU(3) ChPT is checked in Fig. 3. For $f_{K}$ the NLO contribution is about $40 \%$ of the LO one almost independent of the up-down quark mass. This implies that the strange quark mass is not small enough to be treated in the SU(3) ChPT up to NLO. However, the extension to NNLO is not a good choice in a practical sense: The NNLO contribution could be $20 \%$ by naive power counting and a full determination of additional LECs is not achieved without increasing the data points significantly. We take a different direction: the SU(2) ChPT fit with the analytical expansion in terms of the strange quark mass about the physical value. 

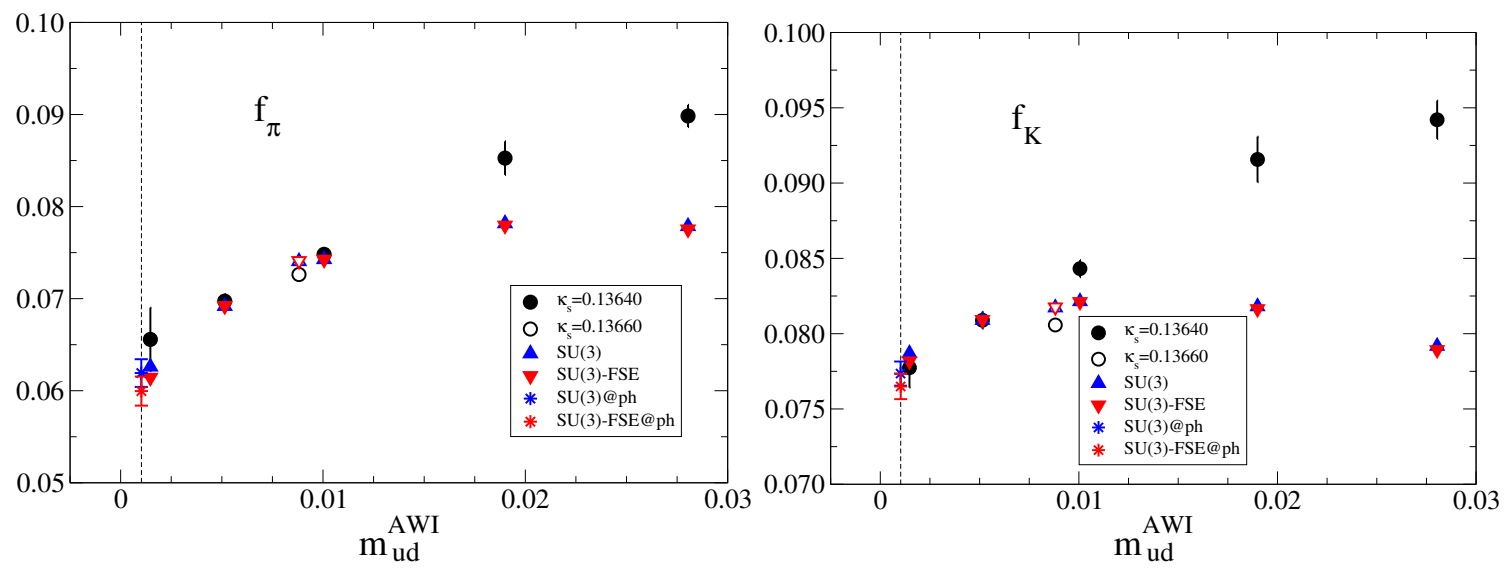

Figure 2: Fit results for $f_{\pi}$ (left) and $f_{K}$ (right). Black symbols represent the lattice results. Red triangles denote the SU(3) ChPT results plotted at the measured quark masses. Open and filled symbols distinguish between the results at $\kappa_{\mathrm{S}}=0.13660$ and 0.13640 . Star symbols represent the extrapolated values at the physical point which is denoted by vertical dotted line.

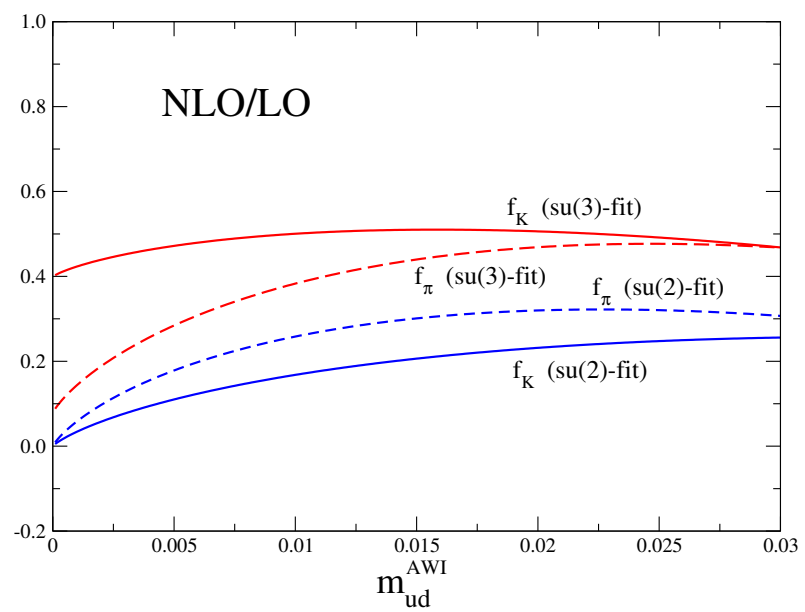

Figure 3: Ratio of the NLO contribution to the LO one in the chiral expansions of the pseudoscalar meson decay constants. Solid (open) lines represent the SU(3) (SU(2)) ChPT case. The strange quark mass is fixed at the physical value.

\subsection{SU(2) ChPT}

We try further chiral analyses on the pseudoscalar meson sector employing the SU(2) ChPT up to NLO, where the $K$ meson is treated as a matter field in the isospin 1/2 linear representation of the SU(2) chiral transformation[36]. The strange quark contributions to the SU(2) LECs are analytically expanded about the physical value. The SU(2) ChPT formulae for $m_{\pi}$ and $f_{\pi}$ are given by

$$
\frac{m_{\pi}^{2}}{2 m_{\mathrm{ud}}}=B\left\{1+\frac{1}{16 \pi^{2}} \frac{\bar{m}_{\pi}^{2}}{f^{2}} \ln \left(\frac{\bar{m}_{\pi}^{2}}{\mu^{2}}\right)+4 \frac{\bar{m}_{\pi}^{2}}{f^{2}} l_{3}\right\}
$$




$$
f_{\pi}=f\left\{1-\frac{1}{8 \pi^{2}} \frac{\bar{m}_{\pi}^{2}}{f^{2}} \ln \left(\frac{\bar{m}_{\pi}^{2}}{\mu^{2}}\right)+2 \frac{\bar{m}_{\pi}^{2}}{f^{2}} l_{4}\right\}
$$

with $\bar{m}_{\pi}^{2}=m_{\mathrm{ud}} B . B$ and $f$ are linearly expanded in terms of the strange quark mass: $B=B_{s}^{(0)}+$ $m_{\mathrm{s}} B_{s}^{(1)}$ and $f=f_{s}^{(0)}+m_{\mathrm{s}} f_{s}^{(1)}$. For $m_{K}$ and $f_{K}$ we employ the following fit formulae:

$$
m_{K}^{2}=\bar{m}_{K}^{2}+\beta_{m} m_{\mathrm{ud}}, \quad f_{K}=\bar{f}\left\{1+\beta_{f} m_{\mathrm{ud}}-\frac{3}{4} \frac{2 B m_{\mathrm{ud}}}{16 \pi^{2} f} \ln \left(\frac{2 B m_{\mathrm{ud}}}{\mu^{2}}\right)\right\},
$$

where $\bar{m}_{K}^{2}$ and $\bar{f}$ are also linearly expanded in terms of the strange quark mass: $\bar{m}_{K}^{2}=\alpha_{m}+\gamma_{m} m_{\mathrm{s}}$ and $\bar{f}=\bar{f}_{s}^{(0)}+m_{\mathrm{s}} \bar{f}_{s}^{(1)}$.
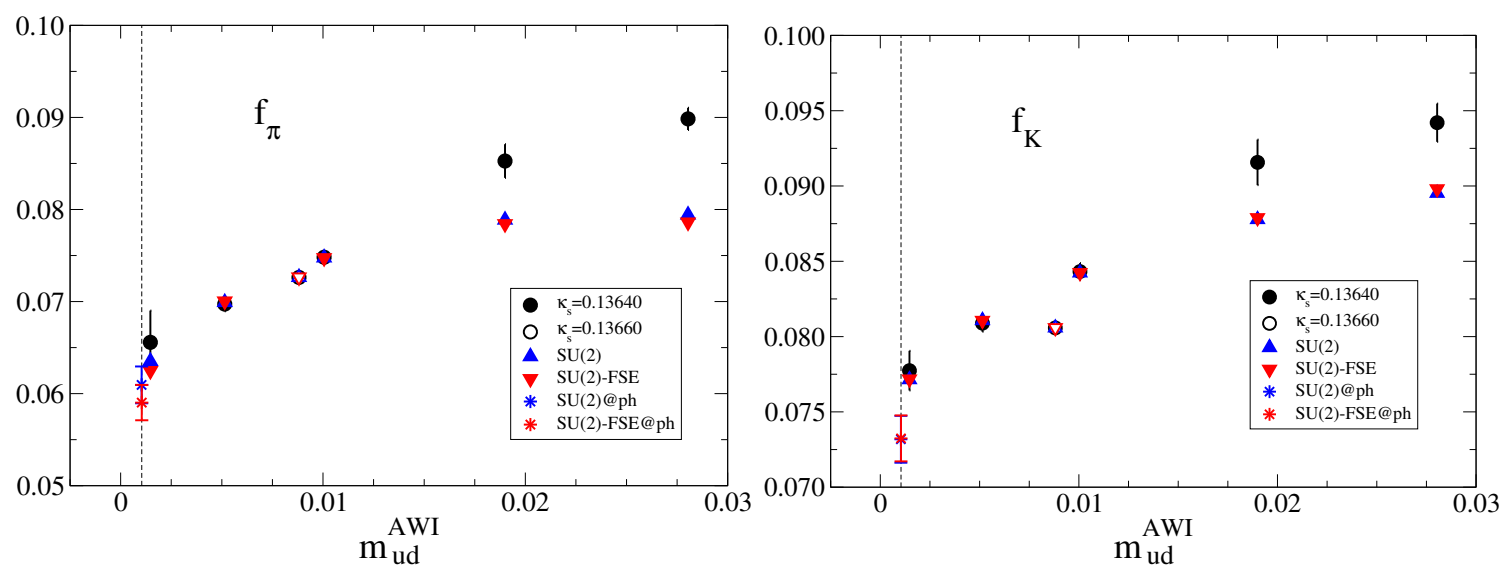

Figure 4: Same as Fig. 2 for the SU(2) ChPT.

A simultaneous fit to $m_{\pi}^{2} / 2 m_{\mathrm{ud}}, f_{\pi}$ and $f_{K}$ leads to the results of $\bar{l}_{3}=3.14(23), \bar{l}_{4}=4.04(19)$ and $\bar{l}_{3}=3.23(21), \bar{l}_{4}=4.10(20)$ with and without the finite size corrections, respectively. We apply an independent fit to $m_{K}^{2}$. The results for $\bar{l}_{3}$ and $\bar{l}_{4}$ are added in Fig. 1, where we find that all the lattice results are consistent except the MILC result of $\bar{l}_{3}$. The fit results for $f_{\pi}$ and $f_{K}$ are depicted in Fig. 4. The lattice data are well described by the SU(2) ChPT combined with the analytic expansion in terms of the strange quark mass. The corresponding $\chi^{2} /$ dof are $0.43(77)$ and $0.33(68)$ with and without the finite size corrections, respectively, which are reasonable magnitude contrary to the SU(3) case. In Fig. 3 we compare the convergence behaviors between the SU(3) and the $\mathrm{SU}(2)$ cases. The ratio of the NLO contribution to the LO one in the SU(2) ChPT is drastically reduced from the SU(3) case in the range of $m_{\mathrm{ud}} \lesssim 0.01$.

\section{ChPT analyses on the baryon sector}

It is intriguing to investigate the quark mass dependence of the baryon mass based on the heavy baryon ChPT (HBChPT). Let us consider the nucleon mass as an example. The SU(2) heavy baryon ChPT formula up to NNLO is given by[37]

$$
m_{N}=m_{0}-4 c_{1} m_{\pi}^{2}-\frac{6 g_{A}^{2}}{32 \pi^{2} f_{\pi}^{2}} m_{\pi}^{3}
$$




$$
\begin{aligned}
& +\left[e_{1}(\mu)-\frac{6}{64 \pi^{2} f_{\pi}^{2}}\left(\frac{g_{A}^{2}}{m_{0}}-\frac{c_{2}}{2}\right)-\frac{6}{32 \pi^{2} f_{\pi}^{2}}\left(\frac{g_{A}^{2}}{m_{0}}-8 c_{1}+c_{2}+4 c_{3}\right) \ln \left(\frac{m_{\pi}}{\mu}\right)\right] m_{\pi}^{4} \\
& +\frac{6 g_{A}^{2}}{256 \pi f_{\pi}^{2} m_{0}^{2}} m_{\pi}^{5}+O\left(m_{\pi}^{6}\right)
\end{aligned}
$$

with $m_{0}, f_{\pi}, c_{1}, c_{2}, c_{3}, g_{A}, e_{1}$ low energy constants. Note that the inclusion of the $O\left(m_{\pi}^{5}\right)$ term, which is obtained by the relativistic baryon ChPT[38], little affects on the following analyses because of its tiny contribution.

The HBChPT fit is performed under several constraints. Firstly, the fit parameters are restricted to $m_{0}, c_{1}, e_{1}$. For a direct comparison with the 2 flavor results of the QCDSF-UKQCD Collaborations[39], we choose $g_{A}=1.267, c_{2}=3.2 \mathrm{GeV}^{-1}$ with $c_{3}=-3.4 \mathrm{GeV}^{-1}$ or $c_{3}=-4.7 \mathrm{GeV}^{-1}$. The former is refereed to as Fit-A and the latter as Fit-B. We employ the value of $f_{\pi}$ at the chiral limit obtained by the SU(2) ChPT fit for $m_{\pi}, f_{\pi}$ and $f_{K}$. Secondly, the ChPT fit is applied to the data series at $\kappa_{\mathrm{s}}=0.13640$ which show a few $\%$ level of variation for $m_{\mathrm{s}}^{\mathrm{AWI}}$. We take two fit ranges: $0.13781 \leq \kappa_{\mathrm{ud}} \leq 0.13727$ for Range-I and $0.13781 \leq \kappa_{\mathrm{ud}} \leq 0.13700$ for Range-II.

Table 3 presents the fit results for the LECs in comparison with the QCDSF/UKQCD results[39]. We also list the ETM results obtained at $\beta=3.9$, which yields similar lattice spacing to ours, with the choice of $c_{3}=-3.45 \mathrm{GeV}^{-1}[40]$. The results show reasonable agreement in case that we take the similar values for $c_{3}$, We also give the nucleon sigma term evaluated through $\sigma_{N \pi}=$ $m_{\pi}^{2}\left(\partial m_{N} / \partial m_{\pi}^{2}\right)$ with the aid of Eq.(4.1). In Fig. 5 we draw the fit results and investigate each contribution of the LO, NLO, NNLO and $O\left(m_{\pi}^{2}\right)$ terms in Eq.(4.1). It is remarkable that the lattice results are well described up to $m_{\pi}^{2} \sim 0.5 \mathrm{GeV}^{2}$ both for Fit-A and Fit-B. We also find reasonable values for $\chi^{2} /$ dof in Table 3. However, this is achieved by a drastic cancellation between the LO and NLO contributions. It is hard to say that the convergence of the SU(2) HBChPT is controlled beyond $m_{\pi}^{2} \sim 0.2 \mathrm{GeV}^{2}$.
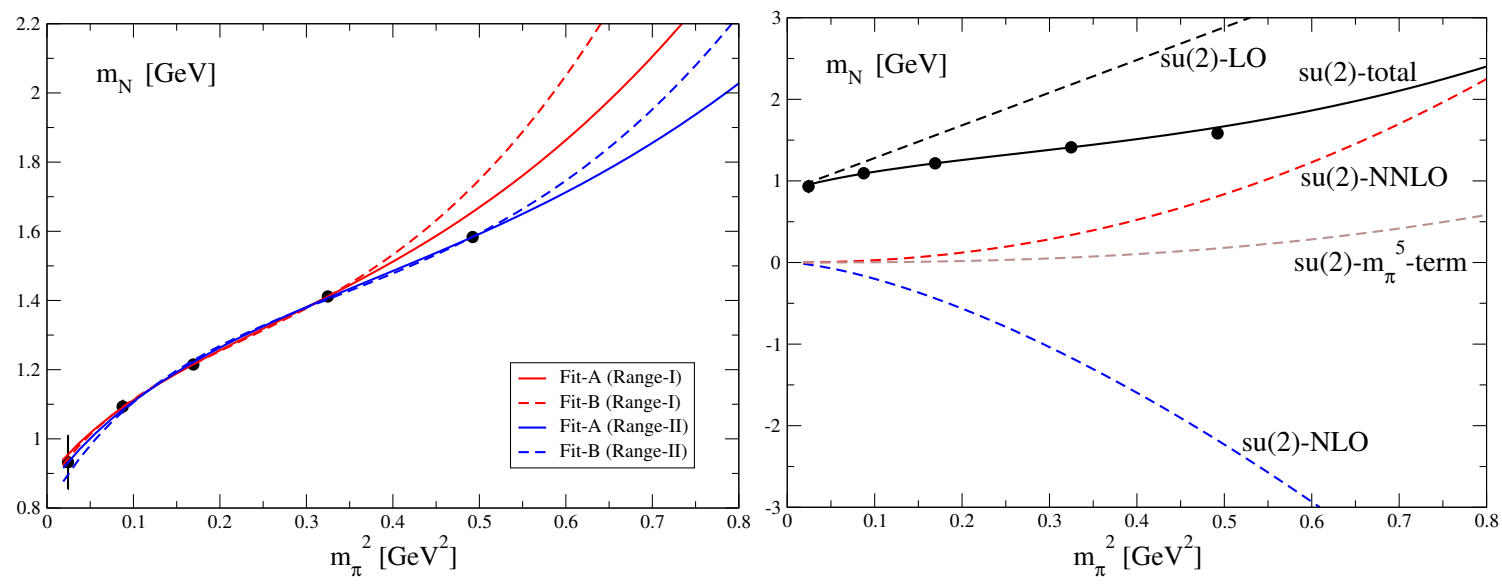

Figure 5: Fit results of the nucleon mass using the SU(2) heavy baryon ChPT formula (left) and each contribution of the LO, NLO, NNLO and $O\left(m_{\pi}^{5}\right)$ terms in the case of Fit-A with Range-I (right). 
Table 3: Fit results for $m_{0}, c_{1}, e_{1}$ in comparison with the QCDSF/UKQCD and the ETM results. Values for the nucleon sigma term are also presented.

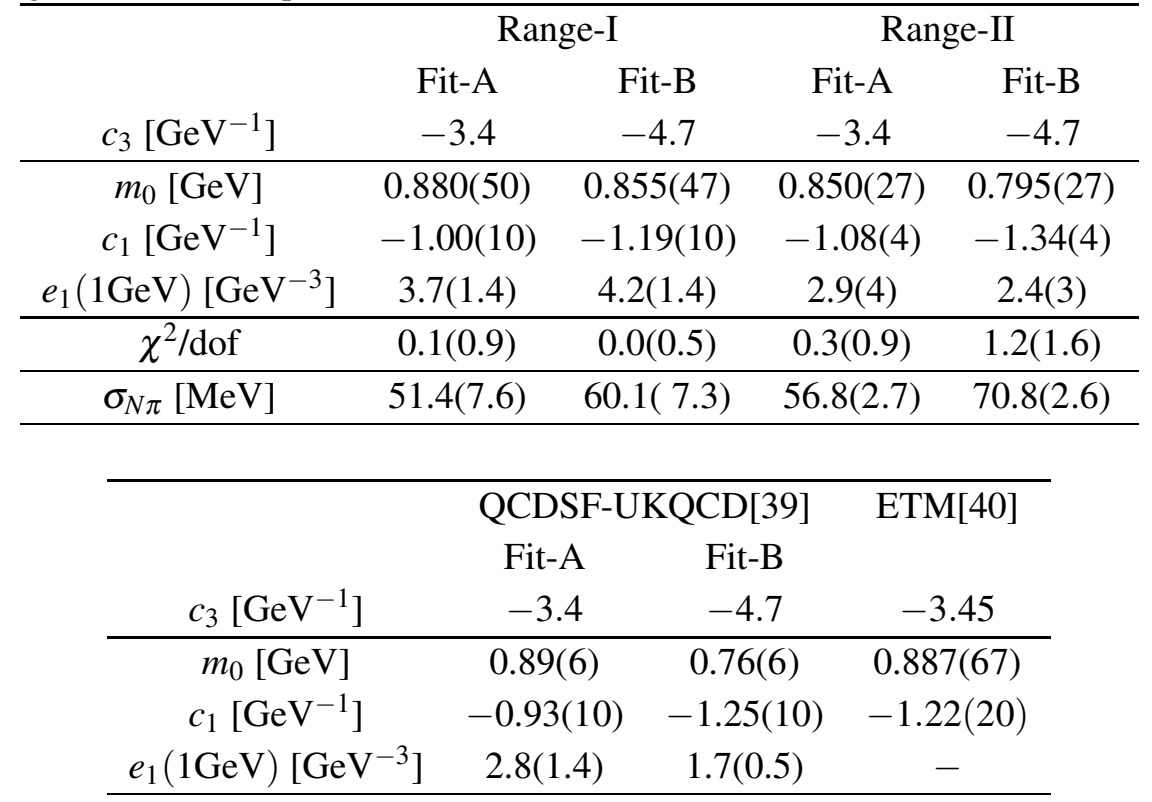

\section{Results at the physical point}

We choose $m_{\pi}, m_{K}$ and $m_{\Omega}$ as physical inputs to determine the up-down and the strange quark masses and the lattice cut-off. The SU(2) ChPT analyses are applied to the quark mass dependences of $m_{\pi}, f_{\pi}$ and $f_{K}$ taking account of the finite size corrections evaluated at the one-loop level. We assume a simple linear quark mass dependences for $m_{K}^{2}$. These points are already explained in Sec. 3.2. We also employ linear forms for the chiral fits of the vector and the baryon masses. It is difficult to rely on the heavy meson effective theory[41] and the heavy baryon ChPT because of their bad convergence behaviors in the chiral expansions[42]. Figure 6 compares the light hadron spectrum at the physical point with the experimental values. The results are encouraging. The largest discrepancy is at most $3 \%$, which may be explained by possible $O\left(\left(a \Lambda_{\mathrm{QCD}}\right)^{2}\right)$ cutoff errors. What we are left with is a proper analysis of the $\rho$ meson and the $\Delta$ baryon as resonance states.

We calculate the bare quark masses using the axial vector Ward-Takahashi identity (AWI) with the nonperturbatively $O(a)$-improved axial vector current[24]. The renormalization factors $Z_{A}$ and $Z_{P}$ are perturbatively evaluated up to one-loop level[25, 26]. In Table 4 we present the results for the quark masses, the lattice cutoff and the pseudoscalar meson decay constants together with the experimental values and the recent $2+1$ flavor lattice QCD results. The physical up-down quark mass is just $30 \%$ smaller than our lightest one $m_{\mathrm{ud}}^{\overline{\mathrm{MS}}}(\mu=1 / a)=3.5 \mathrm{MeV}$ at $\left(\kappa_{\mathrm{ud}}, \kappa_{\mathrm{s}}\right)=$ $(0.13781,0.13640)$. We find that the RBC/UKQCD results for the physical quark masses give rather larger values than ours. This may be caused by different renormalization procedures: a nonperturbative implementation of the RI-MOM scheme for the former and one-loop perturbation for the latter. In fact, the ratio of the up-down quark mass to the strange one, which should be independent of the renormalization factors, shows a good agreement. It may be also suggestive that 


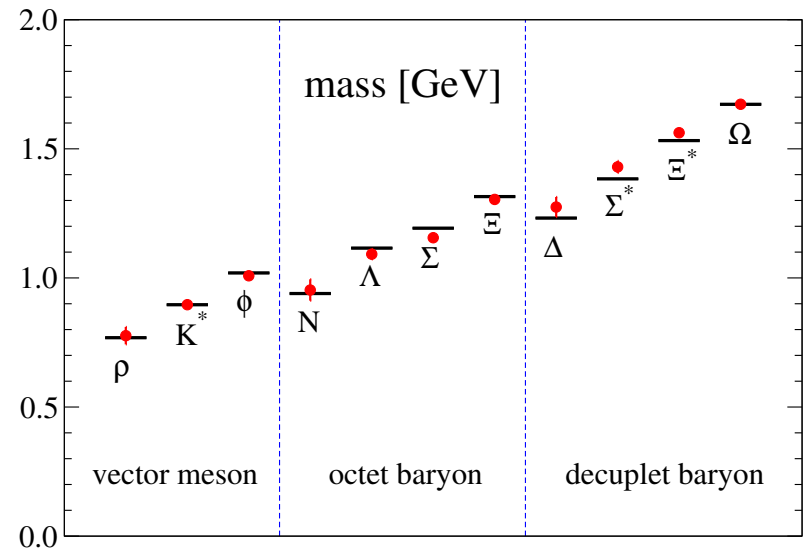

Figure 6: Light hadron spectrum extrapolated to the physical point (red circles) in comparison with the experimental values (black bars).

the MILC results obtained with two-loop renormalization factors are found in the middle of ours and the RBC/UKQCD ones. We are now evaluating the nonperturbative renormalization factors employing the Schrödinger functional scheme.

In Table 4 we also present the results for the pseudoscalar meson decay constants at the physical point employing the perturbative renormalization factor at one-loop level. We find that our results are consistent with the experimental values within the errors. On the other hand, the $\mathrm{RBC} / \mathrm{UKQCD}$ results are smaller than the experimental values by $5 \%$, albeit rather large systematic uncertainties are estimated. Since the MILC results are free from the uncertainties due to the finite renormalization, they use $f_{\pi}$ as a physical input.

Table 4: Cutoff, renormalized quark masses, pseudoscalar meson decay constants determined with $m_{\pi}, m_{K}$ and $m_{\Omega}$ as physical inputs. Quark masses are renormalized at $2 \mathrm{GeV}$.

\begin{tabular}{|c|c|c|c|c|c|}
\hline & \multicolumn{2}{|c|}{ physical point } & \multirow[t]{2}{*}{ experiment[43] } & \multirow[t]{2}{*}{ RBC/UKQCD[29] } & \multirow[t]{2}{*}{ MILC[30] } \\
\hline & w/o FSE & w/ FSE & & & \\
\hline$a^{-1}[\mathrm{GeV}]$ & $2.176(31)$ & $2.176(31)$ & - & $1.729(28)$ & continuum \\
\hline$m_{\mathrm{ud}}^{\overline{\mathrm{MS}}}[\mathrm{MeV}]$ & $2.509(46)$ & $2.527(47)$ & - & $3.72(16)(33)(18)$ & $3.2(0)(1)(2)(0)$ \\
\hline$m_{\mathrm{s}}^{\frac{\mathrm{u}}{\mathrm{MS}}}[\mathrm{MeV}]$ & $72.74(78)$ & $72.72(78)$ & - & $107.3(4.4)(9.7)(4.9)$ & $88(0)(3)(4)(0)$ \\
\hline$m_{\mathrm{s}} / m_{\mathrm{ud}}$ & $29.0(4)$ & $28.8(4)$ & - & $28.8(0.4)(1.6)$ & $27.2(1)(3)(0)(0)$ \\
\hline$f_{\pi}[\mathrm{MeV}]$ & $132.6(4.5)$ & $134.0(4.2)$ & $130.7 \pm 0.1 \pm 0.36$ & $124.1(3.6)(6.9)$ & input \\
\hline$f_{K}[\mathrm{MeV}]$ & 159.2(3.2) & $159.4(3.1)$ & $159.8 \pm 1.4 \pm 0.44$ & $149.6(3.6)(6.3)$ & $156.5(0.4)\left({ }_{-2.7}^{+1.0}\right)$ \\
\hline$f_{K} / f_{\pi}$ & $1.201(22)$ & $1.189(20)$ & $1.223(12)$ & $1.205(18)(62)$ & $1.197(3)\left({ }_{-13}^{+6}\right)$ \\
\hline
\end{tabular}

\section{Results of the physical point simulation}

A direct simulation at the physical point is attempted by choosing the hopping parameters as $\left(\kappa_{\mathrm{ud}}, \kappa_{\mathrm{s}}\right)=(0.137785,0.13660)$, which is estimated using the four data set with $\kappa_{\text {ud }} \geq 0.13754$. 


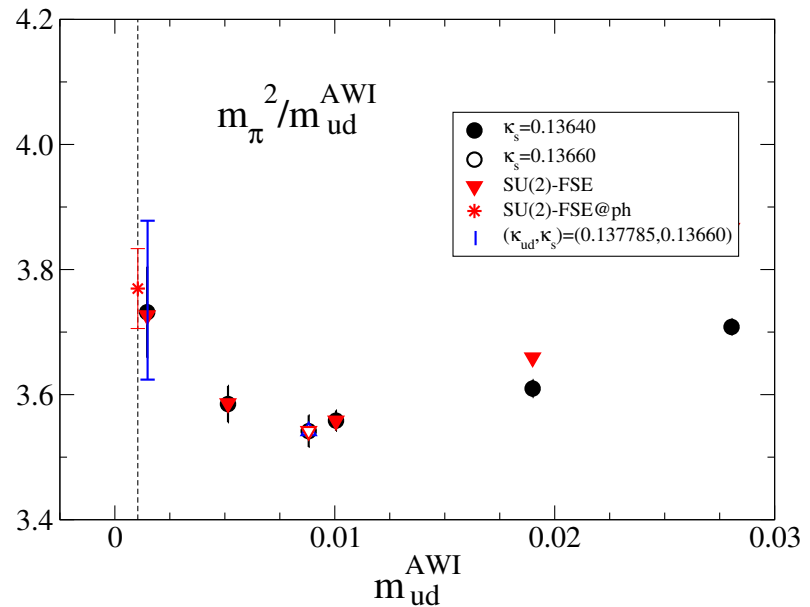

Figure 7: Chiral extrapolation of $m_{\pi}^{2} / m_{\mathrm{ud}}^{\mathrm{AWI}}$ together with the result at $\left(\kappa_{\mathrm{ud}}, \kappa_{\mathrm{s}}\right)=(0.137785,0.13660)$. $\mathrm{SU}(2) \mathrm{ChPT}$ fit is applied to four data points with $m_{\mathrm{ud}}^{\mathrm{AWI}} \lesssim 0.01$.

The measured up-down and the strange quark masses are

$$
m_{\mathrm{ud}}^{\overline{\mathrm{MS}}}(\mu=2 \mathrm{GeV})=3.59(20) \mathrm{MeV}, \quad m_{\mathrm{s}}^{\overline{\mathrm{MS}}}(\mu=2 \mathrm{GeV})=73.72(44) \mathrm{MeV},
$$

where $a^{-1}$ is determined from $m_{\Omega}$ at $\left(\kappa_{\mathrm{ud}}, \kappa_{\mathrm{s}}\right)=(0.137785,0.13660)$. The strange quark mass is successfully tuned at the physical value given in Table 4. Although the up-down quark mass is $5 \sigma$ away from the physical value, it is shown that the chiral corrections due to the deviation are negligibly small or comparable to the current statistical errors. We discuss the importance of the physical point simulation from a view point of controlling the systematic errors.

We first investigate the pseudoscalar meson sector. In Fig. 7 we plot $m_{\pi}^{2} / m_{\mathrm{ud}}^{\mathrm{AWI}}$ as a function of $m_{\pi}^{2} / m_{\mathrm{ud}}^{\mathrm{AWI}}$ together with the fit results with the SU(2) ChPT. The result at $\left(\kappa_{\mathrm{ud}}, \kappa_{\mathrm{s}}\right)=$ $(0.137785,0.13660)$ shows an agreement with the extrapolated value at the physical point (red star) within rather large statistical error. Figure 8 illustrates an importance of the physical point simulation. For $f_{\pi}$ we observe that the extrapolated values at the physical point with various fit formulae are consistent within the error bars. The story is different for $f_{K}$ : The extrapolated values are scattered beyond the error bars. This difference is caused by the typical magnitude of the error bar: $4 \%$ for $f_{\pi}$ and $1-2 \%$ for $f_{K}$. The increase of the resolution with the diminishing statistical errors reveals the discrepancies between the various chiral extrapolations. Figure 8 tells us that these uncertainties should be removed by a direct simulation at the physical point. In the pseudoscalar meson sector it is clear that the up-down quark mass at $\left(\kappa_{\mathrm{ud}}, \kappa_{\mathrm{s}}\right)=(0.137785,0.13660)$ is so close to the physical value that the chiral corrections from the physical point are not relevant within the current statistical errors.

Since we employ $m_{\Omega}$ as a physical input to determine the lattice cutoff, it is intriguing to check the result of $m_{\Omega}$ at $\left(\kappa_{\mathrm{ud}}, \kappa_{\mathrm{s}}\right)=(0.137785,0.13660)$. In Fig. 9 we compare it with the extrapolated value at the physical point. We find a good agreement between them. Moreover, the result at $\left(\kappa_{\mathrm{ud}}, \kappa_{\mathrm{s}}\right)=(0.137785,0.13660)$ has a smaller error than the extrapolated value. In this case the chiral corrections from the the physical point could be comparable to the statistical error. It is 

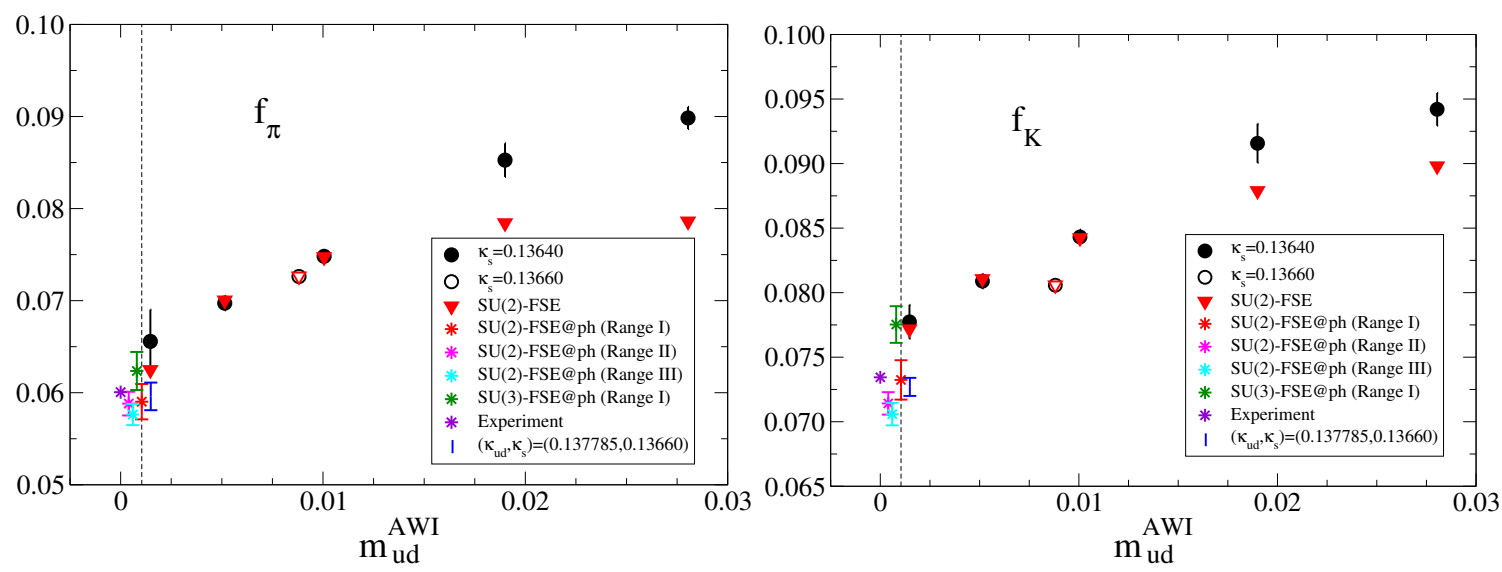

Figure 8: Chiral extrapolations of $f_{\pi}$ (left) and $f_{K}$ (right) together with the results at $\left(\kappa_{\mathrm{ud}}, \kappa_{\mathrm{s}}\right)=$ $(0.137785,0.13660)$. Red symbols represent the SU(2) ChPT fit applied to four data points with $m_{\mathrm{ud}}^{\mathrm{AWI}} \lesssim 0.01$.

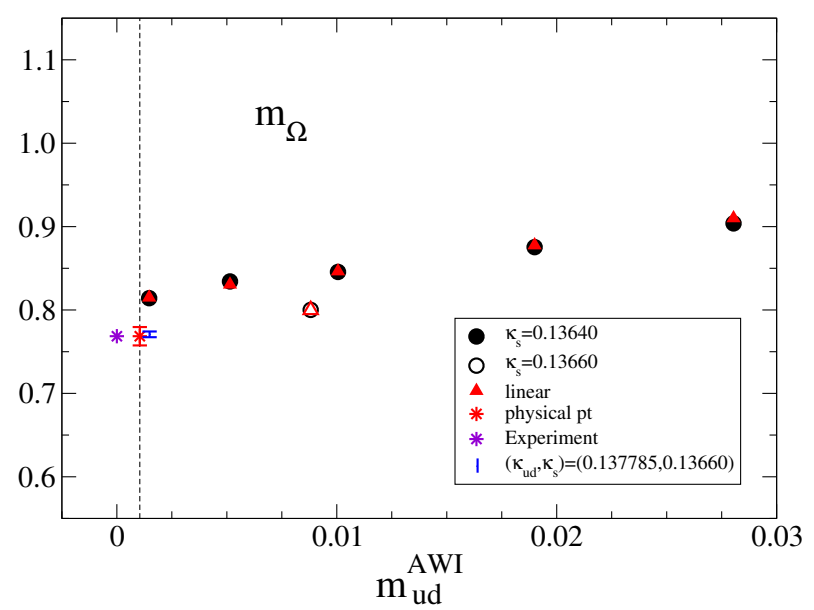

Figure 9: Chiral extrapolation of $m_{\Omega}$ together with the result at $\left(\kappa_{\mathrm{ud}}, \kappa_{\mathrm{s}}\right)=(0.137785,0.13660)$. Linear fit is applied to four data points with $m_{\mathrm{ud}}^{\mathrm{AWI}} \lesssim 0.01$.

fascinating that we are allowed to make a precise measurement of the physical input directly at the physical point.

For the vector channel we compare the results for $m_{K^{*}}$ and $m_{\phi}$ at $\left(\kappa_{\mathrm{ud}}, \kappa_{\mathrm{s}}\right)=(0.137785,0.13660)$ with the extrapolated values in Fig. 10. The situations are similar to the $m_{\Omega}$ case: The extrapolated values are confirmed by the direct measurements at $\left(\kappa_{\mathrm{ud}}, \kappa_{\mathrm{s}}\right)=(0.137785,0.13660)$ with the smaller statistical errors.

\section{Acknowledgment}

Numerical calculations for the present work have been carried out on the PACS-CS computer under the "Interdisciplinary Computational Science Program" of Center for Computational Sciences, University of Tsukuba. This work is supported in part by Grants-in-Aid for Scientific Re- 

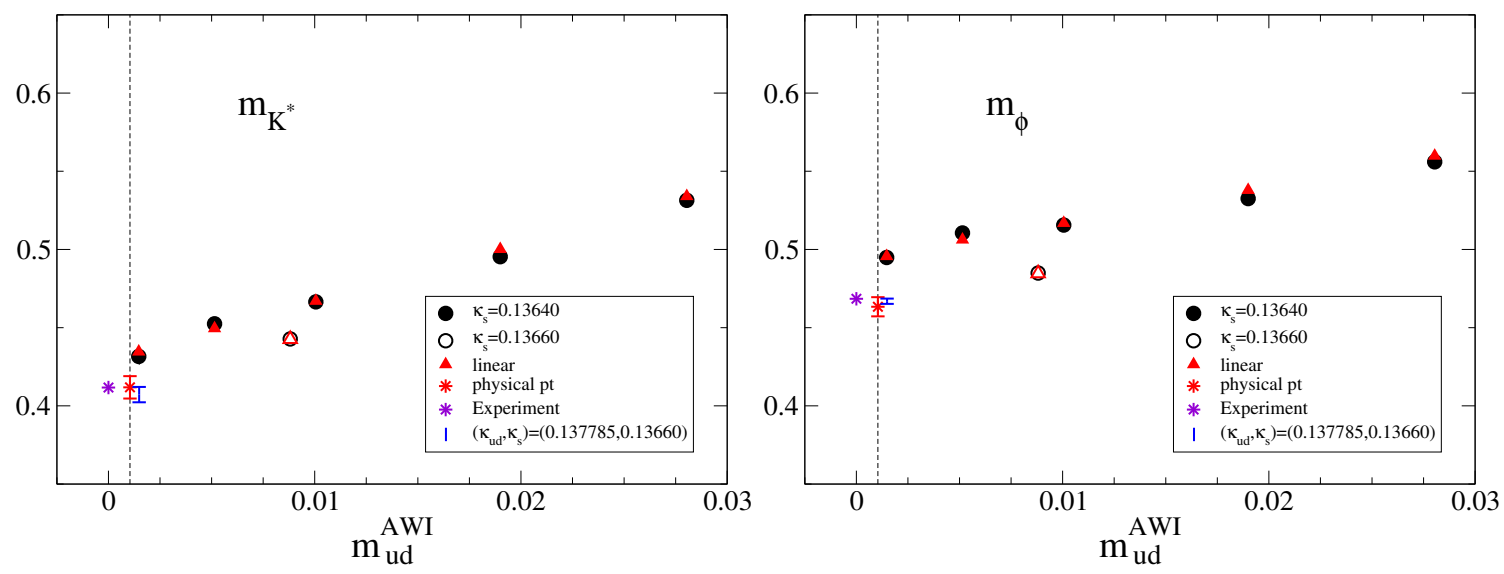

Figure 10: Same as Fig. 9 for $m_{K^{*}}$ (left) and $m_{\phi}$ (right).

search from the Ministry of Education, Culture, Sports, Science and Technology (Nos. 16740147, 17340066, 18104005, 18540250, 18740130, 19740134, 20340047, 20540248, 20740123, 20740139 ) .

\section{References}

[1] CP-PACS and JLQCD Collaborations, T. Ishikawa et al., Phys. Rev. D78, 011502 (2008).

[2] JLQCD Collaboration, S. Aoki et al., Phys. Rev. D65, 094507 (2002).

[3] CP-PACS and JLQCD Collaborations, S. Aoki et al., Phys. Rev. D73, 034501 (2006).

[4] Y. Iwasaki, preprint, UTHEP-118 (Dec. 1983), unpublished.

[5] PACS-CS Collaboration, S. Aoki et al., PoS LAT2005, 111 (2005).

[6] PACS-CS Collaboration, A. Ukawa et al., PoS LAT2006, 039 (2006).

[7] T. Boku et al., Proceedings of CCGRID 2006, pp.233 (2006).

[8] PACS-CS Collaboration, K-I. Ishikawa et al. PoS LAT2006, 027 (2006).

[9] Y. Kuramashi, PoS LATTICE2007, 017 (2007).

[10] PACS-CS Collaboration, N. Ukita et al., PoS LATTICE 2007, 138 (2007).

[11] PACS-CS Collaboration, D. Kadoh et al., PoS LATTICE 2007, 109 (2007).

[12] S. Aoki et al., arXiv:0807.1661 [hep-lat].

[13] PACS-CS Collaboration, N. Ukita et al., PoS LATTICE 2008, 097 (2008).

[14] PACS-CS Collaboration, D. Kadoh et al., PoS LATTICE 2008, 092 (2008).

[15] M. Lüscher, JHEP 0305, 052 (2003); Comput. Phys. Commun. 165, 199 (2005).

[16] J. C. Sexton and D. H. Weingarten, Nucl. Phys. B380, 665 (1992).

[17] A. Kennedy, Nucl. Phys. B (Proc. Suppl.) 140, 190 (2005).

[18] M. Hasenbusch, Phys. Lett. B519, 177 (2001). 
[19] M. Hasenbusch and K. Jansen, Nucl. Phys. B659, 299 (2003).

[20] R. Brower, T. Ivanenko, A. Levi and K. Orginos, Nucl. Phys. B484, 353 (1997).

[21] M. Parks et al., SIAM J. Sci. Comput. 28, 1651 (2006).

[22] S. Aoki, O. Bär, T. Ishikawa and S. Takeda, Phys. Rev. D73, 014511 (2006); S. Takeda, Wilson chiral perturbation theory for $2+1$ flavors, Doctor Thesis (unpublised).

[23] J. Gasser and H. Leutwyler, Nucl. Phys. B250, 465 (1985).

[24] CP-PACS/JLQCD and ALPHA Collaborations, T. Kaneko et al., JHEP 0704, 092 (2007).

[25] S. Aoki et al., Phys. Rev. D58, 074505 (1998).

[26] Y. Taniguchi and A. Ukawa, Phys. Rev. D58, 114503 (1998).

[27] G. Colangelo, S. Dürr and C. Haefeli, Nucl. Phys. B721, 136 (2005).

[28] G. Amorós, J. Bijnens and P. Talavera, Nucl. Phys. B602, 87 (2001).

[29] RBC and UKQCD Collaborations, C. Allton et al., arXiv:0804.0473[hep-lat].

[30] C. Bernard et al., arXiv:0710.1118[hep-lat].

[31] J. Gasser and H. Leutwyler, Ann of Phys. 158, 142 (1984).

[32] G. Colangelo, J. Gasser and H. Leutwyler, Nucl. Phys. B603, 125 (2001).

[33] L. Del Debbio et al., JHEP 0702, 056 (2007); JHEP 0702, 082 (2007).

[34] C. Urbach, PoS LATTICE2007, 022 (2007).

[35] JLQCD Collaborations, J. Noaki et al., arXiv:0806.0894[hep-lat].

[36] A. Roessl, Nucl. Phys. B555, 507 (1999).

[37] J. Gasser, M. E. Sainio and A. Svarc, Nucl. Phys. B 307, 779 (1988).

[38] M. Procura, T. R. Hemmert and W. Weise, Phys. Rev. D 69, 034505 (2004).

[39] QCDSF-UKQCD Collaboration, A. Ali Khan et al., Nucl. Phys. B 689, 175 (2004).

[40] European Twisted Mass Collaboration, C. Alexandrou et al.,Phys. Rev. D78, 014509 (2008).

[41] E. Jenkins, A. Manohar and M. Wise, Phys. Rev. Lett. 75, 2272 (1995).

[42] B. Borasoy and U.-G. Meissner, Phys. Lett. B365, 285 (1996); J. Bijnens, P. Gosdzinsky and P. Talavera, Nucl. Phys. B501, 495 (1997).

[43] W.-M. Yao et al., J. Phys. G33, 1 (2006). 